\title{
Supporting Information: Single-Particle Tracking of Human Lipoproteins
}

\author{
Michel de Messieres, ${ }^{\dagger}$ Abby Ng, ${ }^{\dagger}$ Cornelio J. Duarte, ${ }^{\ddagger}$ Alan T. Remaley, ${ }^{\ddagger}$ and Jennifer C. Lee ${ }^{\dagger}$ \\ ${ }^{\dagger}$ Laboratory of Molecular Biophysics and ${ }^{\ddagger}$ Lipoprotein Metabolism Section, National Heart, Lung, and Blood \\ Institute, National Institutes of Health, Bethesda, Maryland 20892
}

\section{Table of Contents}

Index of Supplementary Files

Protocols

Protocol S1. Chamber Construction

Protocol S2. Labeling Lipoproteins with Alexa Fluor Dyes

Protocol S3. Image Analysis

Protocol S4. Transmission Electron Microscopy

Protocol S5. Sample Preparation for Chambers

Protocol S6. Example Particle Tracks

\section{$\underline{\text { Tables }}$}

Table S1. Dilutions of Lipoproteins

Table S2. BCA Assay for Lipoproteins

Figures

Figure S1. Confinement Chamber

Figure S2. UV Absorption Measurements

Figure S3. Donor A Dominant Fluorescent Signals

Figure S4. Donor B Dominant Fluorescent Signals

Figure S5. Donor C Dominant Fluorescent Signals

Figure S6. Donor A Fluorescent Signal Scatter Plots

Figure S7. Donor B Fluorescent Signal Scatter Plots

Figure S8. Donor C Fluorescent Signal Scatter Plots

Figure S9. Donor A TEM Images

Figure S10. Donor B TEM Images

Figure S11. Donor C TEM Images

Figure S12. Dynamic Light Scattering (DLS)

Figure S13. Simulated Data

\section{$\underline{\text { References }}$}




\section{Index of Supplementary Files}

- Donor A Original.avi - Movie of first 20 frames for one Donor A data set

- Donor A Corrected.avi - Movie after background subtraction

- Donor A Particle Tracks - Example particles from movie

- Donor B Original.avi - Movie of first 20 frames for one Donor B data set

- Donor B Corrected.avi - Movie after background subtraction

- Donor B Particle Tracks - Example particles from movie

- Donor C Original.avi - Movie of first 20 frames for one Donor C data set

- Donor C Corrected.avi - Movie after background subtraction

- Donor C Particle Tracks - Example particles from movie

- Simulated Original.avi - Movie of first 20 frames from one Simulated data set

- Simulated Corrected.avi - Movie after background subtraction

- Simulated Particle Tracks - Example particles from movie 


\section{Protocols}

\section{Protocol S1. Chamber Construction}

\section{Parts:}

- PEG-coated glass slide (Microsurfaces PEG_02)

- $22 \times 40$ mm PEG-coated coverslip (Microsurfaces PEG_02 custom order)

- Double-sided 1/2" width tape (Scotch ${ }^{\circledR}$ 3M 34-8507-8235-9)

- $\quad$ 2" Spring Clamp (Home Depot SKU 573682)

1. PEG-coated glass slides and PEG-coated $22 \times 40 \mathrm{~mm}$ coverslips were ordered from Microsurfaces, Inc, packed in sets of 5 . On arrival we stored each pack at $-80^{\circ} \mathrm{C}$. We allowed packs to reach room temperature for about 15 minutes before opening them to avoid condensation forming on the surface of the slides. Upon opening each pack, we transferred each slide and coverslip (using clean tweezers) to individual containers (saved from previous shipments). The coverslips were placed between two layers of tissue paper with an additional ball of tissue paper on top to prevent the coverslip from sliding. We then placed each container in a small zip-lock plastic bag, pumped nitrogen into the container, sealed the container, then pumped nitrogen into the bag, and sealed it. The bags were then frozen at $-80{ }^{\circ} \mathrm{C}$. Before opening the individual packs, we again allowed them to reach RT to avoid condensation.

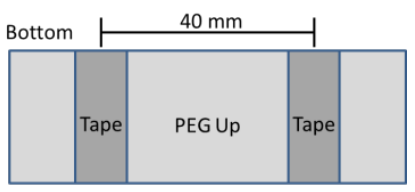

Side

Side

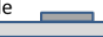

2. Setup a PEG-coated glass slide with PEG face up on a surface which is clean and suitable for cutting (we used the back of a plastic $1.5 \mathrm{~mL}$ test tube rack). Place double-sided tape across glass and extending beyond the sides with $40 \mathrm{~mm}$ spacing. Cut away excess tape as shown. The tape may have defects which should be avoided.

3. Keep the glass slide clean (in a pipette box) while removing a PEG-coated $22 \times 40 \mathrm{~mm}$ coverslip from its individual container, using clean tweezers. Apply a strong stream of nitrogen to both PEG-coated surfaces to remove any residual dust. Proceed to step 4 quickly to minimize the chance of any dust falling on the slide.

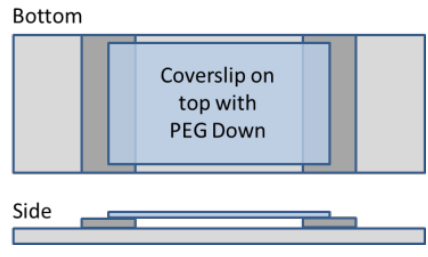

4. Place the PEG-coated $22 \times 40 \mathrm{~mm}$ coverslip (PEG down) on the glass slide (PEG up), aligned on the tape as shown. Using the bottom tip of a plastic test tube (Eppendorf $1.5 \mathrm{~mL}$ ), rub down on the coverslip where it touches the tape to make a uniform seal. Air bubbles trapped under the tape can usually be rubbed out. Defects or insufficient pressure may cause the slide to detach from the tape before the chamber is complete. 


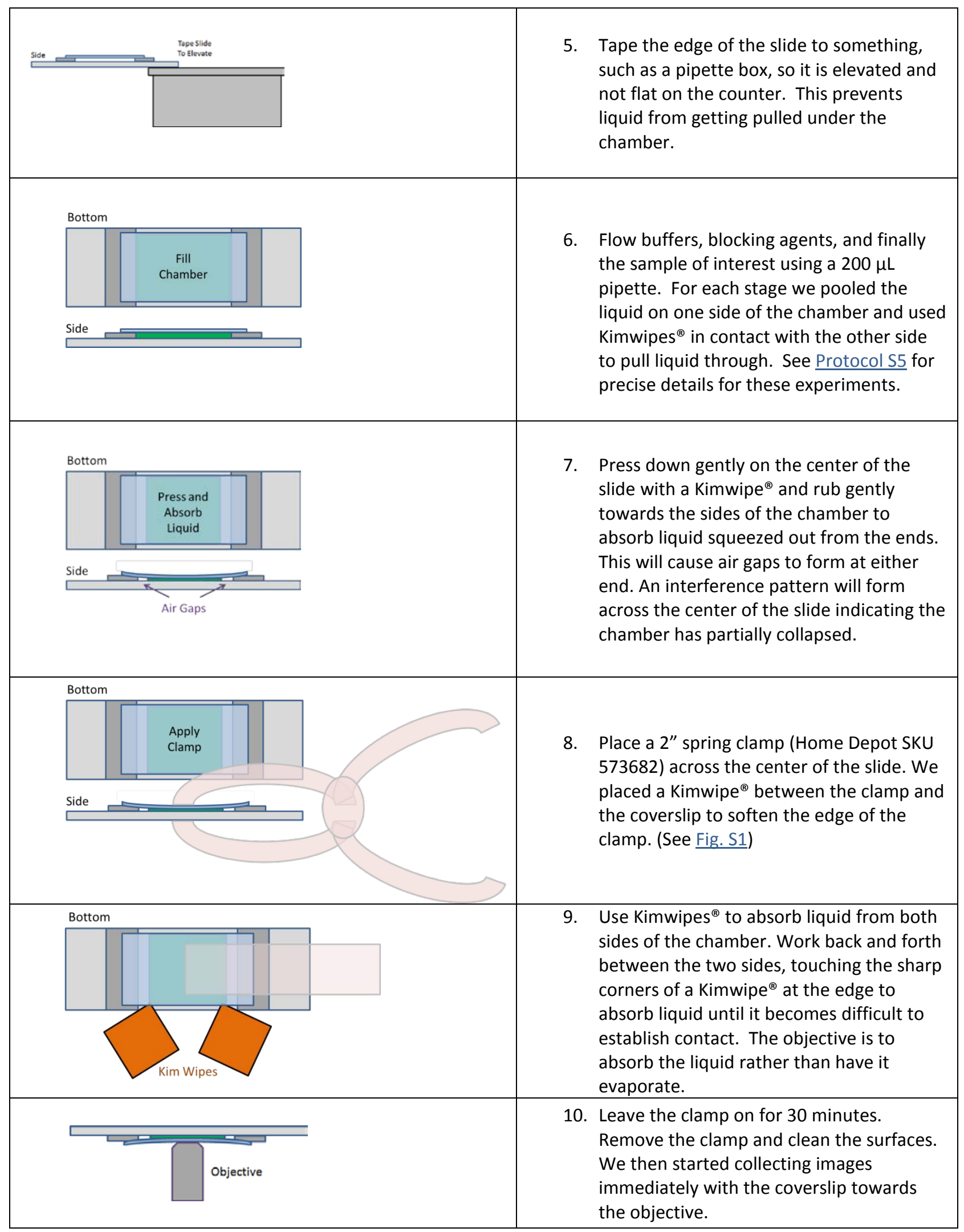




\section{Protocol S2. Labeling Lipoproteins with Alexa Fluor ${ }^{\circledR}$ Dyes}

Lipoproteins from human blood were separated by stepwise density gradient ultracentrifugation using $\mathrm{KBr}$ as previously described ${ }^{[1]}$. Individual fractions of $\mathrm{HDL}(d=1.063-1.21 \mathrm{~g} / \mathrm{mL}), \mathrm{LDL}(d=1.019-1.063$ $\mathrm{g} / \mathrm{mL})$, and VLDL $(d=0.95-1.006 \mathrm{~g} / \mathrm{mL})$ were dialyzed in PBS to remove $\mathrm{KBr}$ and had a protein concentration of order $1 \mathrm{mg} / \mathrm{mL}$ but this varied for the 3 donors used in this study (Table S2). All individual experiments were done with HDL, LDL, and VLDL isolated from the same donor. Blood was collected from normal patients at the NIH Clinical Center as approved by NIH NHLBI IRB.

Primary amine groups on VLDL, LDL, and HDL were labeled with Alexa Fluor ${ }^{\circledast}$ fluorophores:

\begin{tabular}{|c|c|c|c|}
\hline Lipoprotein & Fluorophore & Company & Catalog \# \\
\hline HDL & Alexa Fluor 488 TFP Ester & Life Technologies & A-37570 \\
\hline LDL & Alexa Fluor 594 NHS Ester & Life Technologies & A-37572 \\
\hline VLDL & Alexa Fluor 647 NHS Ester & Life Technologies & A-37573 \\
\hline
\end{tabular}

We had a general expectation that VLDL and LDL would be predominantly labeled on ApoB100 while HDL would be labeled on ApoAl. However, other proteins are present and may also serve as labeling sites.

The protocol for labeling lipoproteins:

Buffer A: $50 \mathrm{mM} \mathrm{NaPi}, 150 \mathrm{mM} \mathrm{NaCl}, \mathrm{pH} 7.4$.

Buffer B: Buffer A with 0.5 mM EDTA.

1) Dilute HDL $1 / 100$ and LDL $1 / 10$ in Buffer A. VLDL is not diluted.

2) Add $100 \mu \mathrm{L}$ of $1 / 100 \mathrm{HDL}, 1 / 10 \mathrm{LDL}$, or $1 / 1 \mathrm{VLDL}$ directly to tubes of $100 \mu \mathrm{g}$ Alexa dye.

3) Mix by pipetting up and down until dye pellet is dissolved.

4) Transfer to a $1.5 \mathrm{~mL}$ Eppendorf tube with a mini stir bar ( $5 \times 2 \mathrm{~mm})$.

5) Stir gently ( $\sim 2$ revolutions per second) $30 \mathrm{~min}$ at RT under a constant nitrogen environment.

6) Add $400 \mu \mathrm{L}$ of Buffer $A$ to each tube.

7) Load the $500 \mu \mathrm{L}$ total solution onto a PD-10 column (GE Healthcare) equilibrated with Buffer $A$.

8) Load $2.5 \mathrm{~mL}$ of Buffer A - this skips the $1^{\text {st }} 500 \mu \mathrm{L}$ that would normally be collected.

9) Collect $1 \mathrm{~mL}$ and load directly onto a $2^{\text {nd }} P D-10$ column equilibrated with Buffer $A$.

10) Load $2 \mathrm{~mL}$ of Buffer A. Note this skips the $1^{\text {st }} 500 \mu \mathrm{L}$ that would normally be collected.

11) Collect $1 \mathrm{~mL}$. This is the final sample which will be diluted before loading into the chamber.

12) Measure UV absorbance to verify labeling and estimate concentrations. 


\section{Protocol S3. Image Analysis}

Collection of Images: Imaging was conducted at RT using a DeltaVision OMX system (GE Healthcare) with independent CMOS cameras for each channel. We used the structured illumination light path which provides the most power. The gratings create a pattern on the images which can sometimes be seen in the raw data files (see included movies). We did not remove the gratings due to practical considerations as the instrument is shared by many users. This pattern is expected to generate additional noise in the measurements but will not bias the measurements, especially since the particles are moving. Simultaneous 5-ms excitation with

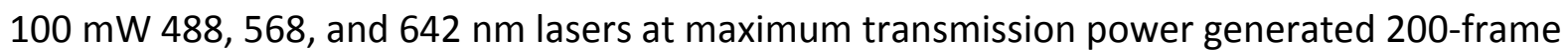
three-channel movies at 200 frames per second ( $1 \mathrm{~s}$ total time) and $512 \times 512$ pixel resolution. We used a TIRF 60x objective and switched to TIRF mode before each acquisition which allowed us to focus only on particles stuck to the bottom coverslip. This may improve the manual focusing since particles on both the top and bottom glass layers will otherwise be visible, but this is not necessary. After focusing, we switched to Structured Illumination mode for acquisition. We used the burst mode and fast camera settings to acquire 200 frames per second ( $5 \mathrm{~ms}$ acquisition time) with negligible latency between successive frames.

Background Subtraction: The images were processed using custom software written in C++. To implement a background subtraction, we fit each pixel value for each of the three channels over time (200 frames) to an exponential decay function. To remove the influence of fluctuations (particles passing over the pixel), we removed all values from the fitting function which were more than 10 standard deviations from the fit. We then repeated the fit with the cutoff set to 8, 6, 4, and then 2 standard deviations, removing points outside the cutoff as we progressed. The resulting exponential fit is subtracted from the raw data. We then applied the same procedure across rows of the image, except using a linear fit instead of an exponential fit.

Note that background subtraction can bias the intensity measurement to lower values if a particle is moving too slowly and falsely interpreted as background. This will have the most effect on the largest particles. This is of less importance for diffusion measurements since this artifact is not expected to change the measured position of the particle. Using a slower frame rate may be more optimal for larger particles.

Camera Alignment: A grid pattern measured simultaneously on all three cameras was used to apply a correction to positions for the red and green channel relative to the blue channel. For the cosmetic purposes of generating movies and representations of the data, we applied the correction to the actual images. However, for the purposes of data analysis we only applied corrections to positions and otherwise conducted all analysis (Gaussian fits and integration) on the original images to avoid pixel rounding complications. 
Determination of Particle Position: To convert particle images into positions we used a 2D Gaussian fit on each channel (red, green, or blue) and used the result with the strongest total intensity. To validate fits, we also conducted Gaussian fits with the initial center at four offset positions (shifted 3 pixels in $x$ and $y$ making four points on a square). A point was only accepted if all four Gaussian fits converged to the same position as the initial fit within 1 pixel error. If a Gaussian fit failed we would manually select new positions on the particle which may then result in an accepted fit. If this did not yield an acceptable fit the frame was excluded.

Determination of Particle Tracks: To determine particle tracks we used a semi-automated routine. First we selected the approximate location of the first frame of a particle and the position was then determined using the Gaussian fit procedure described above. Subsequent frames were automatically predicted as the nearest bright spot to the prior frame and accepted one at a time by manual input. If two particles passed over each other and subsequently could not be identified uniquely, we stopped the particle track on the last frame they could be resolved. Frames that could not pass the Gaussian fit test described above or were too close to the border were excluded. Frames where the particle appeared stuck to the surface were excluded.

Determination of Radius from Particle Tracks: For each particle track, all pairs of valid positions which were separated by one frame were used to determine the effective radius by diffusion, as described in the manuscript. We calculated the radius using step sizes in both the $x$-and $y$ axes and used the average for represented data. Figure 3 and Table 1 in the manuscript show the distribution of radii for the three donors.

For each donor, we calculated mean radius and standard deviation. For HDL and LDL, this was calculated using a Gaussian fit to a histogram of the measured values, minimizing the effect of outliers. For VLDL, a Gaussian does not fit well due to the extended distribution, so we just reported the mean and standard deviation of all values.

Determination of Particle Intensity: While particle location was determined using a Gaussian fit to the fluorescence signal, we did not use the Gaussian fit to determine intensity. We calculated total intensity as the sum of all pixels within an 11 pixel radius. We determined a local background level as the mean of all pixels in the region defined as more than 11 pixel radius and less than 16 pixel radius from the center. The integrated value with background subtracted determines the y axis, labeled as Signal (a.u.), for the included example data sets. Inspection of Signal relative to Time assisted us to identify errors such as particles crossing over each other or a particle becoming stuck to one of the surfaces. 


\section{Protocol S4. Transmission Electron Microscopy}

Radii distributions for HDL, LDL, and VLDL from transmission electron microscope images (Fig. 3 and Table 1 in the manuscript) were produced by imaging the lipoproteins on a JEOL JEM 1200 EX electron microscope (JEOL USA) with an AMT XR-60 6 megapixel digital camera (Advanced Microscopy Techniques) on glow discharged 400 mesh Formvar/carbon grids (Electron Microscopy Sciences). We incubated $5 \mu \mathrm{L}$ of the labeled lipoprotein (using the final fraction from Protocol S2. Labeling Lipoproteins with Alexa Fluor Dyes) for 30 seconds on the grid. In all cases, five drops of $1 \% \mathrm{w} / \mathrm{v}$ uranyl acetate were allowed to fall on and immediately off the grid with a rate of approximately 1-2 drops per second. The stain was then wicked off slowly and allowed to dry. Lipoproteins were imaged at 50,000x magnification. Using ImageJ (v 1.49d), individual lipoproteins were manually marked using the oval selection tool. The corresponding area $(A)$ was calculated and radius $(r)$ was estimated from $A=\pi r^{2}$. Examples of the image analysis are shown for each donor (Fig. S9-S11).

Note on Donor B: The $L D L$ concentrations of Donor B were too low after labeling so in that case we diluted each unlabeled lipoprotein fraction 1/10 in NaPi $(50 \mathrm{mM}), \mathrm{NaCl}(150 \mathrm{mM})$, and $5 \mathrm{mM}$ EDTA followed by incubation at RT for $1 \mathrm{~h}$. HDL for Donor B was still too concentrated so we diluted an additional 1/100 in PBS pH 7.4 immediately before applying to the grid to obtain the images shown in Fig. S10.

Note on EDTA: Lipoproteins sometimes appeared stuck together on the grid and we experimented with using EDTA as we thought the sticking could be inherent to the sample. However, we did not find that EDTA improved the situation and we hypothesize that the lipoproteins may clump as the uranyl acetate dries. Therefore, EM may not be useful for the purpose of demonstrating whether or not some lipoproteins are forming higher order complexes in solution.

\section{Protocol S5. Sample Preparation for Chambers}

1) Construct chamber as described in Protocol S1. Chamber Construction.

2) Flow $200 \mu \mathrm{L}$ of Buffer $B(50 \mathrm{mM} \mathrm{NaPi}, 150 \mathrm{mM} \mathrm{NaCl}, 0.5 \mathrm{mM}$ EDTA, pH 7.4)

3) Flow $1 \mathrm{~mL}$ of $0.1 \mathrm{mg} / \mathrm{mL}$ BSA (Sigma Alderich A2153) in Buffer B Note: This may help reduce sticking with protein experiments but did not seem to reduce lipoprotein sticking.

4) Incubate the BSA for 30 minutes at RT.

5) Flow $2 \mathrm{~mL}$ of Buffer $B$ to wash out BSA.

6) Mix lipoproteins in Buffer B (Our experiments used the dilutions in Table S1).

7) Load in the confinement chamber and clamp as described in Protocol S1. Chamber Construction. 


\section{Protocol S6. Example Particle Tracks}

The example particle tracks are included as supplementary files and represent the tracks for the first 20 lipoproteins in the first movie of each data set. Top left for each image shows the particle track superimposed on the original image of the first frame, as well as the scale bar and identification of the particle type. The gallery (middle) shows the first 60 frames of the particle. Frames which could not be fit to a Gaussian, were too close to the edge, overlapped other particles, or appeared stuck were not included. These frames will appear as gaps in the intensity graph (right middle) which plots all three channels for each particle relative to time. In general each particle will have one dominant channel (red, green, or blue) which identifies the lipoprotein type (HDL, LDL, or VLDL respectively).

The histogram of step sizes (top right) shows measurements made on the two independent axes ( $x$ and $y$ ) and the resulting effective radius and uncertainty determined using the standard equations of diffusion, provided in the manuscript. We also show the radius determined by fitting the histogram ${ }^{[2]}$ rather than simply using mean squared displacement. For this work, we did not use the histogram fits for radius calculations. The histogram fit is an effective means to remove steps which represent stuck particles. Some tracks, especially for $\mathrm{HDL}$, will have insufficient data to obtain good fits for the histogram. We removed frames where particles appeared to be stuck by visual inspection.

The gallery of images shows the first 60 frames of the lipoprotein. In some cases, less than 60 frames were obtained due to the particle becoming stuck, leaving the screen, or passing near another similar particle.

The gallery of profiles has a one-to-one correspondence with the gallery of images. For each track we applied the same scaling to all three channels based on the linear fit to the fluorescence, preserving the relative strength of the three signals. The scaling was applied to best represent each track since some had strong fluorescence and some were very weak. The profile in the $x$-axis direction was generated by binning columns (10 pixels high, 1 pixel wide) across the center of the particle. We did not bin radially because we wanted the noise of values near the middle of the particle to be comparable to values near the edge. Each profile was rendered such that the calculated background level (determined for each track point using a ring of pixels around each particle) is aligned with the bottom of the image, so this provides a visual confirmation that the background level is correct. We also did the same calculation in the $y$-axis direction, so each channel shows two graphs.

The intensity values determined for each track point are represented by summing all pixels which fit in a circle of the same diameter as the represented images or profiles. 


\section{Tables}

Table S1. Dilutions of Lipoproteins. Values given here indicate the dilution of the final labeled lipoprotein fraction collected from the PD-10 column before mixing in the chamber. Each blood donor was different so the concentrations used in the chamber sometimes had to be adjusted. An optimal concentration of particles is not too crowded or too sparse. We recommend using the UV absorption after labeling (Fig. S2) as a rough guide to estimate the initial concentrations.

\begin{tabular}{|c|c|c|c|}
\hline $\begin{array}{c}\text { Measurement } \\
\text { Date }\end{array}$ & Donor & Lipoprotein & Dilution \\
\hline \multirow{3}{*}{$5 / 15 / 15$} & \multirow{2}{*}{$5 / 4 / 15$} & $\mathrm{HDL}$ & 0.033 \\
\cline { 3 - 4 } & & $\mathrm{LDL}$ & 0.167 \\
\cline { 3 - 4 } & \multirow{2}{*}{$6 / 10 / 15$} & $\mathrm{BLDL}$ & 0.050 \\
\cline { 3 - 4 } & $6 / 1 / 15$ & $\mathrm{HDL}$ & \\
\cline { 3 - 4 } & & $\mathrm{LDL}$ & 0.062 \\
\hline & \multirow{2}{*}{$\mathrm{C}$} & $\mathrm{VLDL}$ & 0.892 \\
\hline \multirow{3}{*}{$7 / 14 / 15$} & $7 / 10 / 15$ & $\mathrm{HDL}$ & 0.045 \\
\cline { 3 - 4 } & & $\mathrm{LDL}$ & 0.025 \\
\hline & & $\mathrm{VLDL}$ & 0.250 \\
\hline
\end{tabular}

Table S2. BCA Assay for Lipoproteins. HDL, LDL, and VLDL were prepared for the BCA protein assay by adding $0.5 \mathrm{mM}$ EDTA and $2 \% \mathrm{SDS}^{[3]}$ to the original fractions and then preparing dilutions from 10 to $100 \%$ with dilution buffer $(0.5 \mathrm{mM}$ EDTA, $2 \%$ SDS, $\mathrm{NaPi}(50 \mathrm{mM}), \mathrm{NaCl}(150 \mathrm{mM}), \mathrm{pH} 7.4)$. BSA standards were also prepared with $2 \%$ SDS in the same buffer from 0.025 to $2 \mathrm{mg} / \mathrm{mL}$. $25 \mu \mathrm{L}$ of each sample was pipetted into a 96-well Corning clear bottom microplate and $200 \mu \mathrm{L}$ of freshly prepared Pierce BCA Protein Assay (Thermo Fisher) was added to each well. The plate was shaken for $30 \mathrm{~s}$ on a bench top shaker, incubated at $37^{\circ} \mathrm{C}$ for $30 \mathrm{~min}$, and then was cooled for approximately $10 \mathrm{~min}$. The measurement's linearity can be improved by carefully pipetting $150 \mu \mathrm{L}$ of each well into a fresh well to remove any bubbles caused by the SDS. We then measured absorbance at $562 \mathrm{~nm}$ with 25 flashes using a Tecan Infinite M200 Pro microplate reader. We converted absorbance to concentration using the regime of the BSA standard curve which was most suitable depending on the concentration of the lipoprotein fraction. The measured values scale reasonably well with UV absorption measurements (Fig. S2).

\begin{tabular}{|c|c|c|}
\hline Donor & Lipoprotein & Protein Concentration $(\mathbf{m g} / \mathbf{m L})$ \\
\hline \multirow{4}{*}{$A$} & HDL & 3.1 \\
\cline { 2 - 3 } & LDL & 2.0 \\
\cline { 2 - 3 } & VLDL & 0.5 \\
\hline \multirow{4}{*}{ B } & HDL & 2.2 \\
\cline { 2 - 3 } & LDL & 0.3 \\
\cline { 2 - 3 } & VLDL & 0.3 \\
\hline \multirow{3}{*}{ C } & HDL & 3.9 \\
\cline { 2 - 3 } & LDL & 1.7 \\
\cline { 2 - 3 } & VLDL & 0.5 \\
\hline
\end{tabular}




\section{Figures}
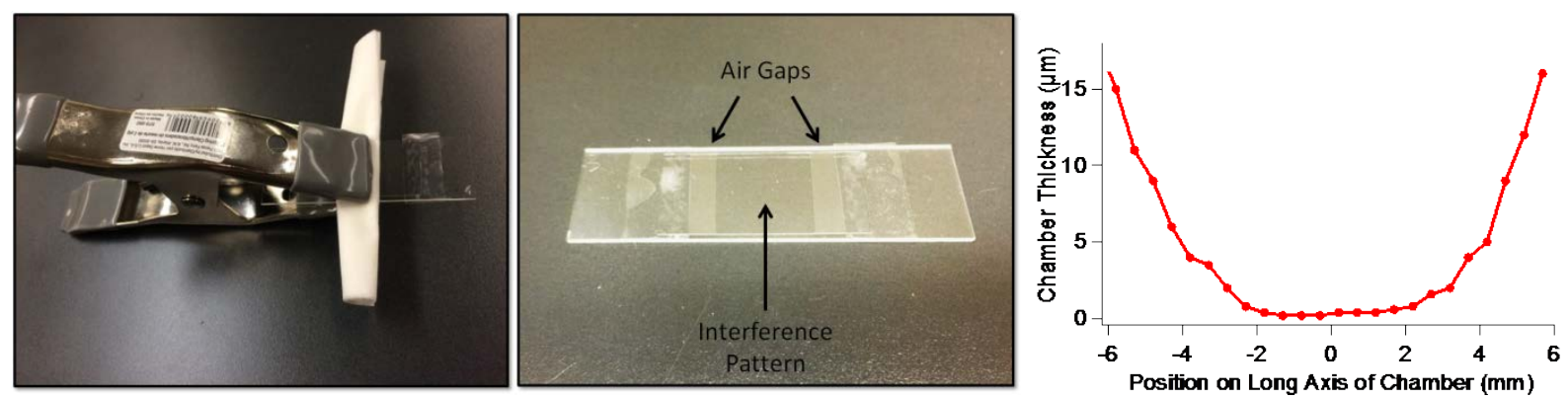

Figure S1. Confinement Chamber. Photo of chamber during construction (left) shows the completed chamber with clamp applied (left). The folded Kimwipe ${ }^{\circledR}$ was used to soften the edge of the clamp. After 30 min, the clamp is removed (middle) and the air gaps on either side persist. A narrow interference band down the middle of the slide (not visible) marks the narrowest point and the ideal imaging location will be slightly shifted from this region. Chamber thickness (right) measured at positions separated by $0.5 \mathrm{~mm}$ along the long axis of the chamber (which is left to right in the middle picture and the $y$ axis in our movies). For each position we moved the stage vertically until the top or bottom of the chamber was visible. This was determined by focusing on particles stuck to the top or bottom or identifying the position where all particles were out of focus. For small values ( $0.2 \mu \mathrm{m}$ or less) the thickness cannot be precisely determined. We did not measure to the edge of the chamber due to physical constraints of the stage. 

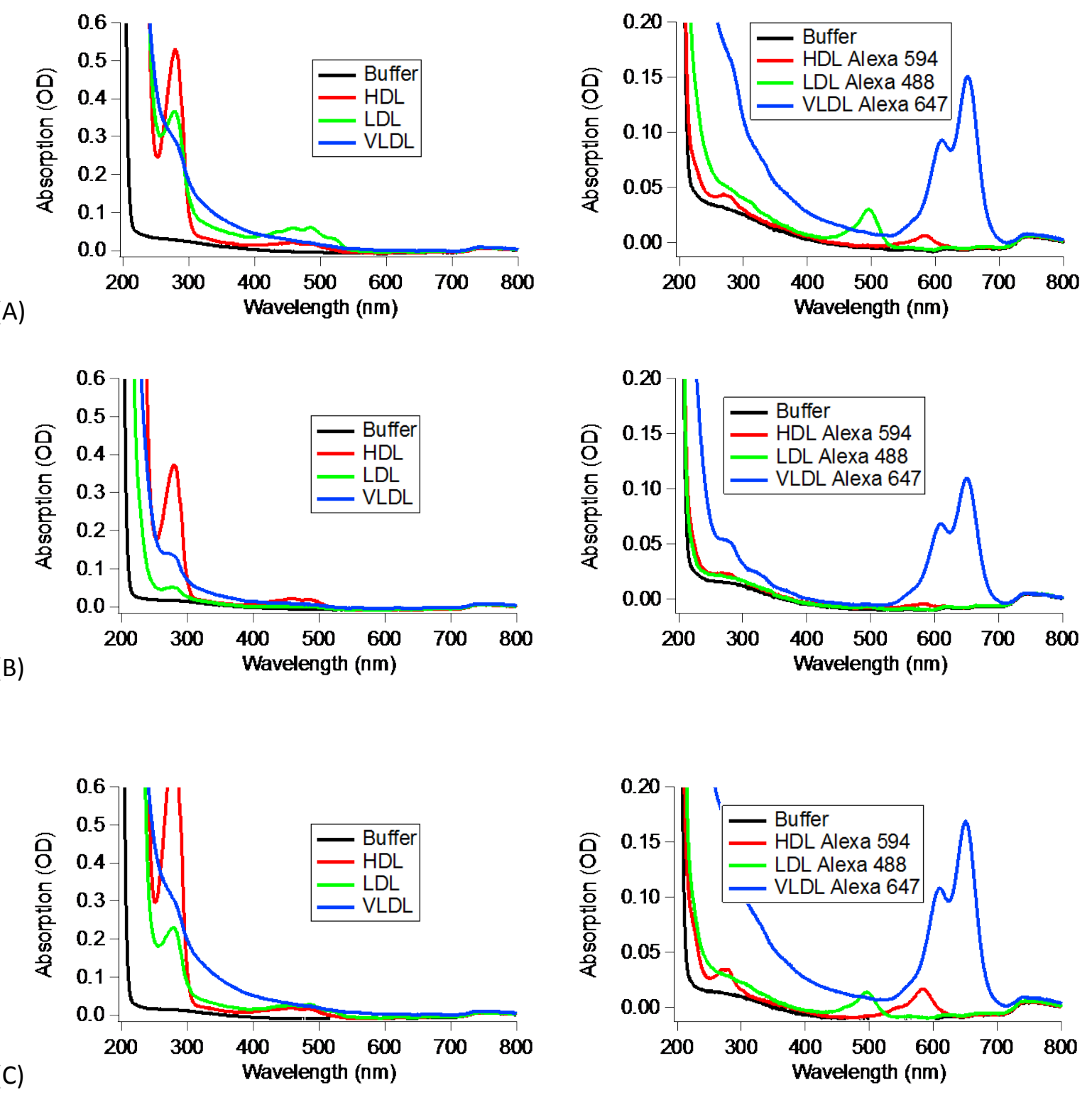

Figure S2. UV Absorption Measurements. UV absorption of HDL (red), LDL (green), and VLDL (blue) diluted 1/10 from original fractions obtained from ultracentrifugation (left) and after labeling with Alexa 594, Alexa 488, and Alexa 647 respectively (right). (A), (B), and (C) indicate Donor A, Donor B, and Donor C respectively. Dilution buffer (black) was $\mathrm{NaPi}(50 \mathrm{mM}), \mathrm{NaCl}$ (150 mM), pH 7.4. Measured using a Cary 300 Bio UV-Visible spectrophotometer in a 1-cm quartz cuvette. 

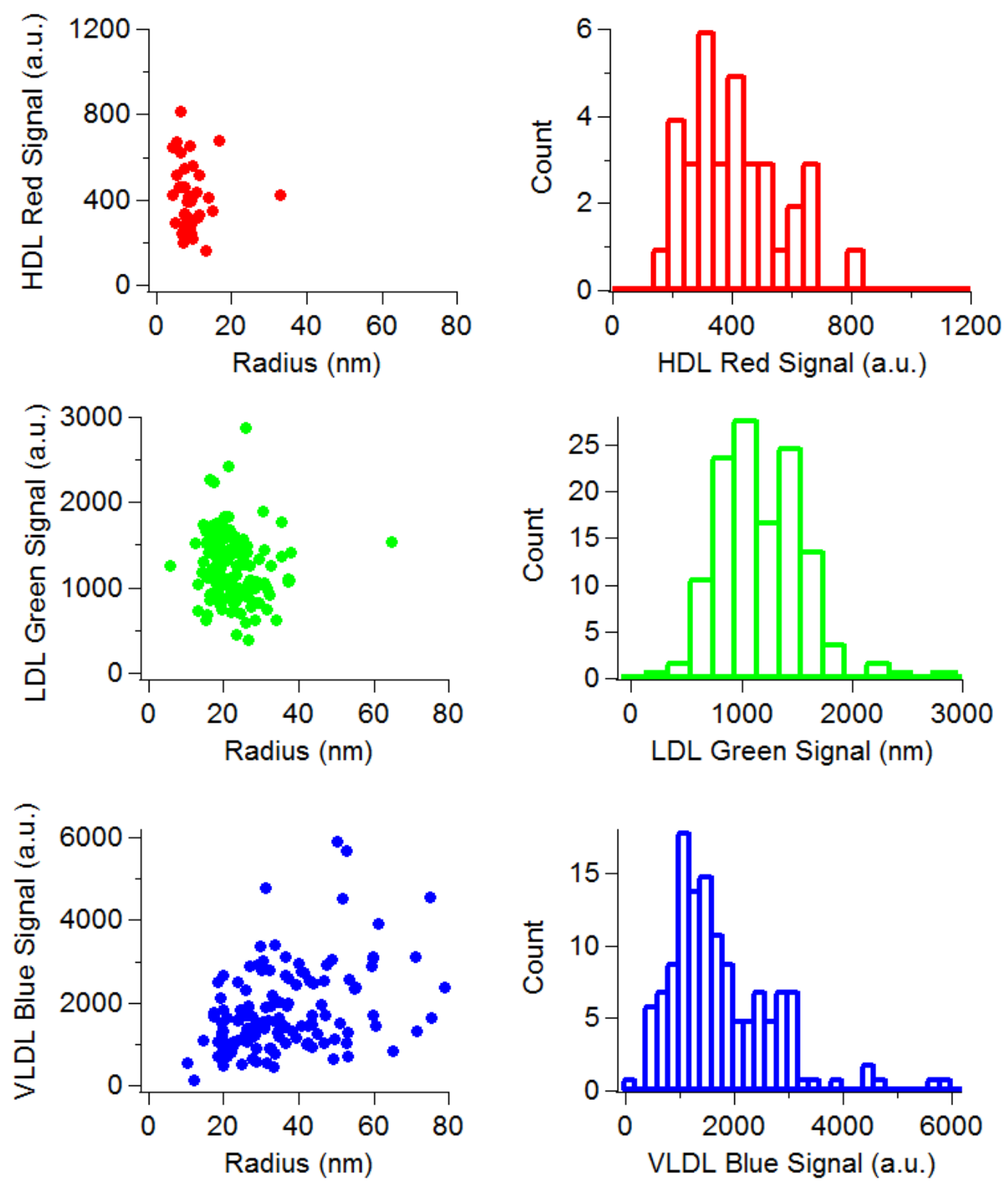

Figure S3. Donor A Dominant Fluorescent Signals. Left shows a scatter plot of the signal relative to radius, determined by diffusion. Right shows a histogram of all measured values. The colors correspond to the channels where red indicates Alexa 594 (HDL), green indicates Alexa 488 (LDL), and blue indicates Alexa 647 (VLDL). 

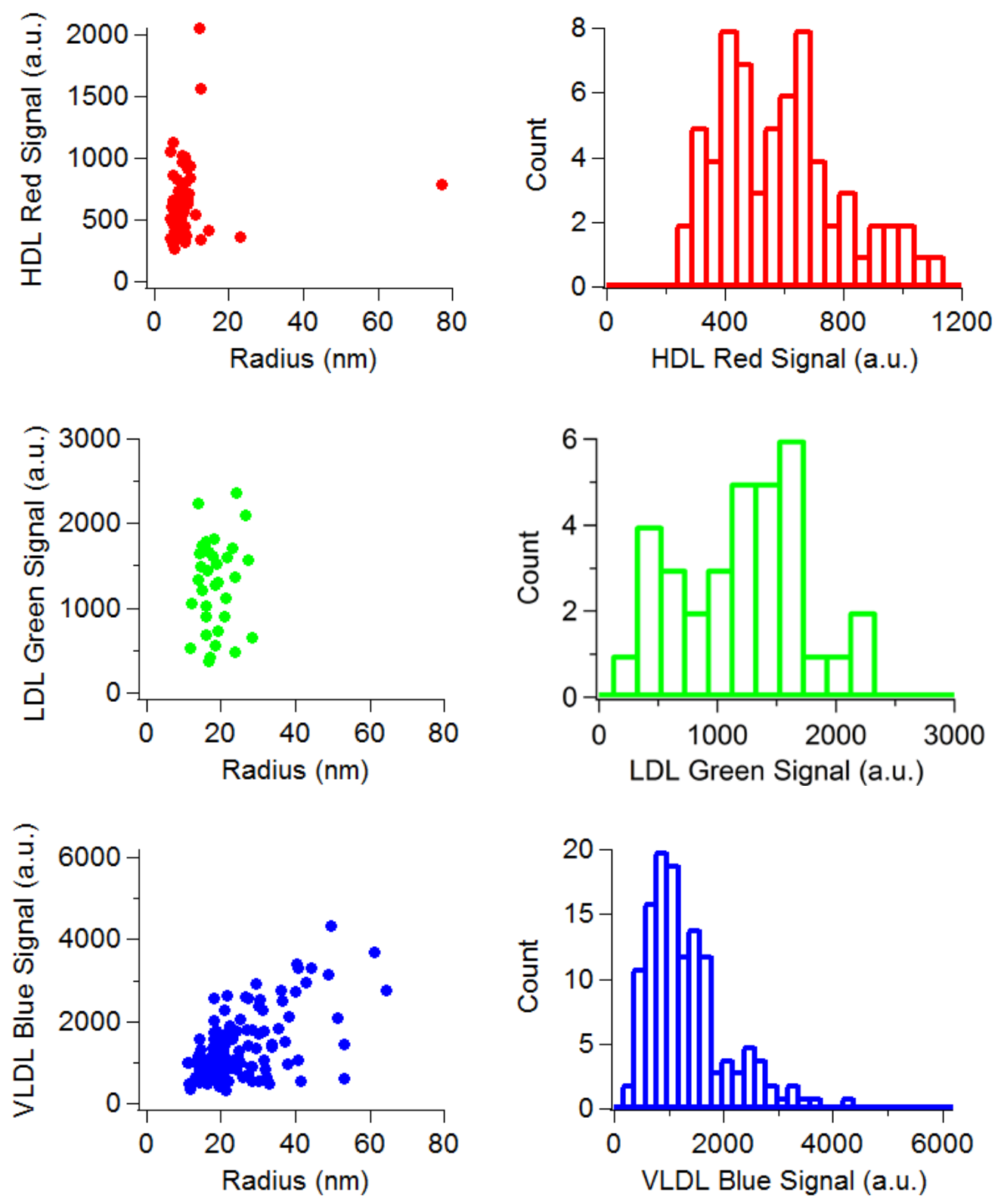

Figure S4. Donor B Dominant Fluorescent Signals. Left shows a scatter plot of the signal relative to radius, determined by diffusion. Right shows a histogram of all measured values. The colors correspond to the channels where red indicates Alexa 594 (HDL), green indicates Alexa 488 (LDL), and blue indicates Alexa 647 (VLDL). 

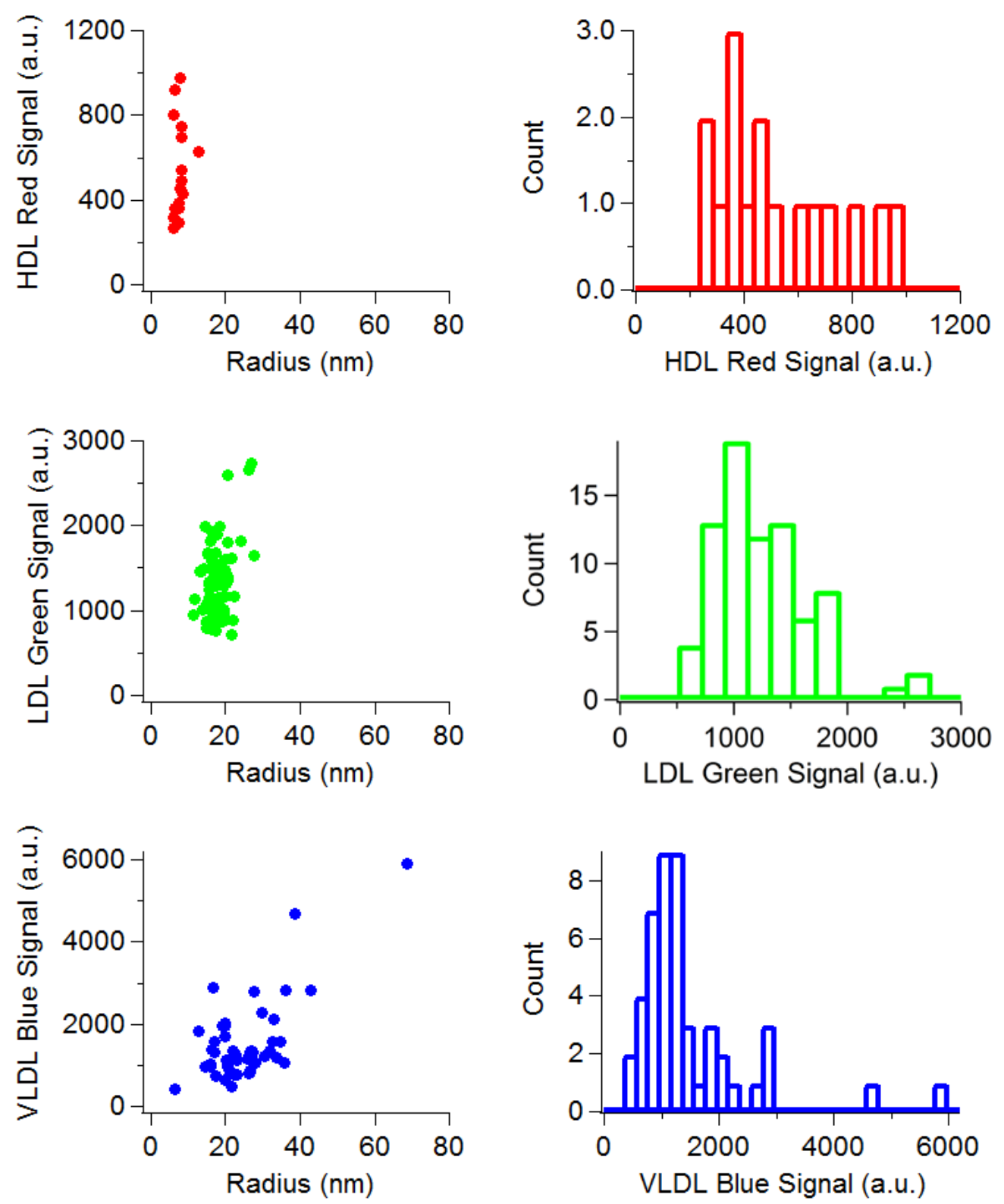

Figure S5. Donor C Dominant Fluorescent Signals. Left shows a scatter plot of the signal relative to radius, determined by diffusion. Right shows a histogram of all measured values. The colors correspond to the channels where red indicates Alexa 594 (HDL), green indicates Alexa 488 (LDL), and blue indicates Alexa 647 (VLDL). 

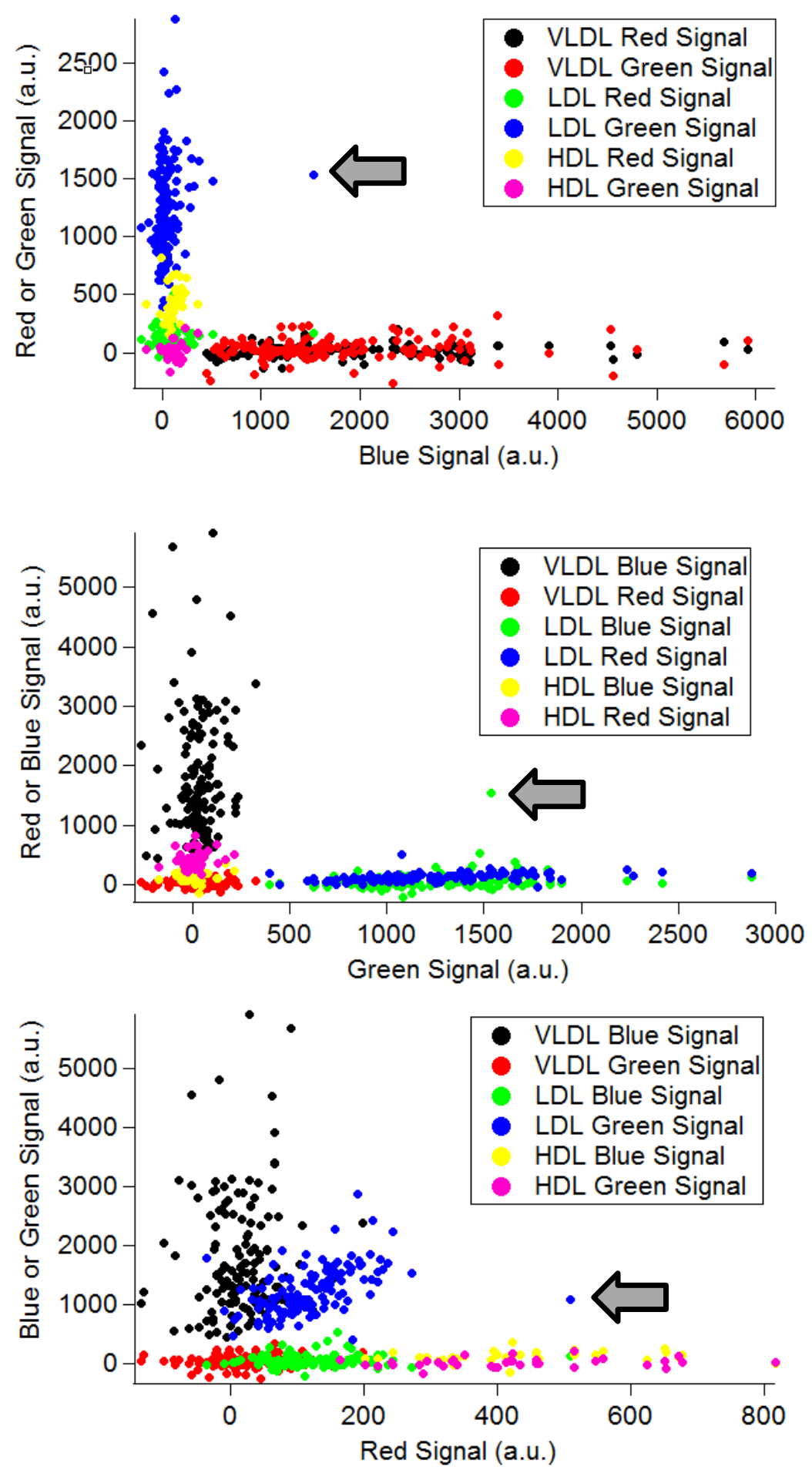

Figure S6. Donor A Fluorescent Signal Scatter Plots. Arrows mark apparent fusion particles. 

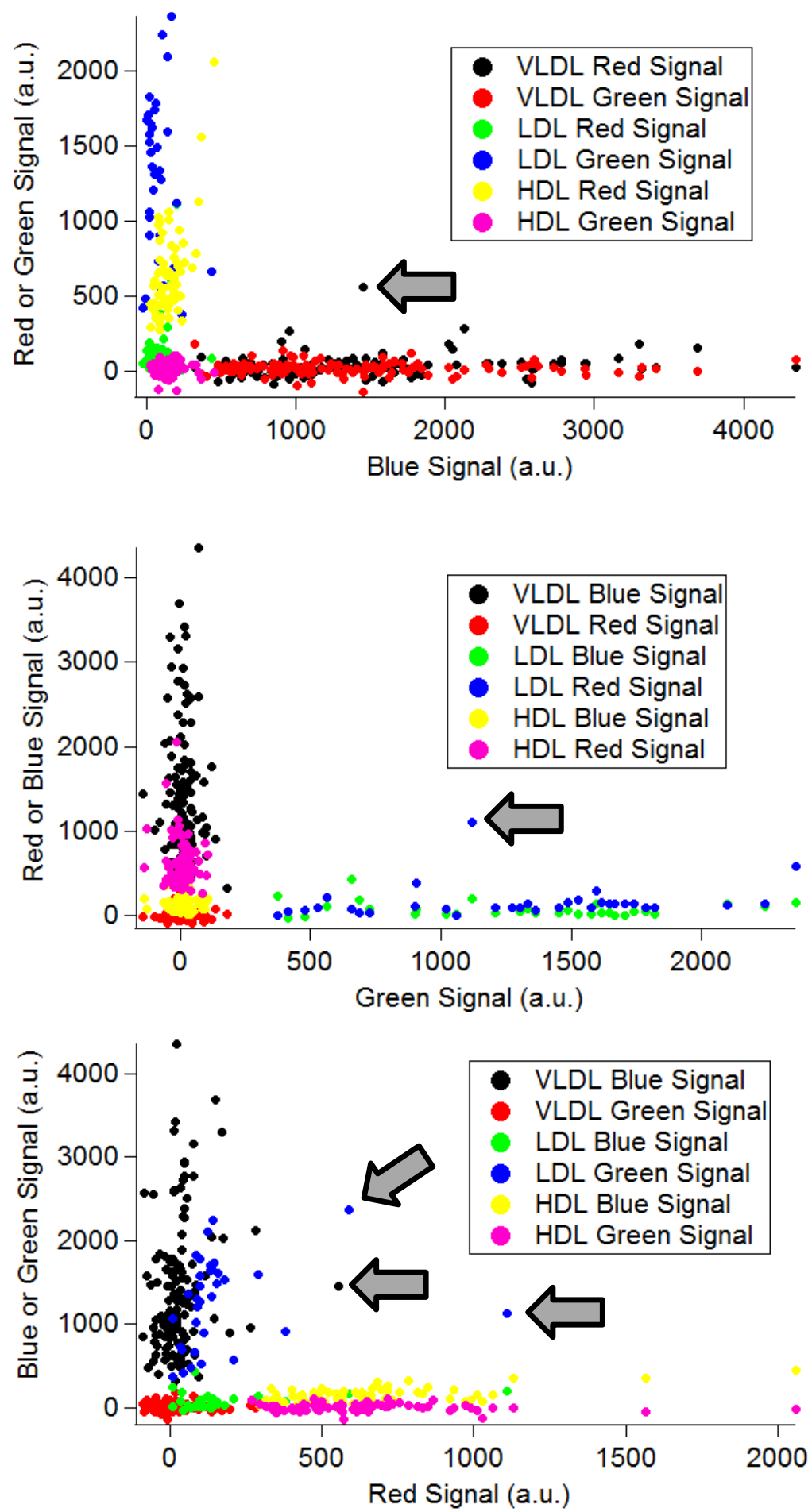

Figure S7. Donor B Fluorescent Signal Scatter Plots. Arrows mark apparent fusion particles. 

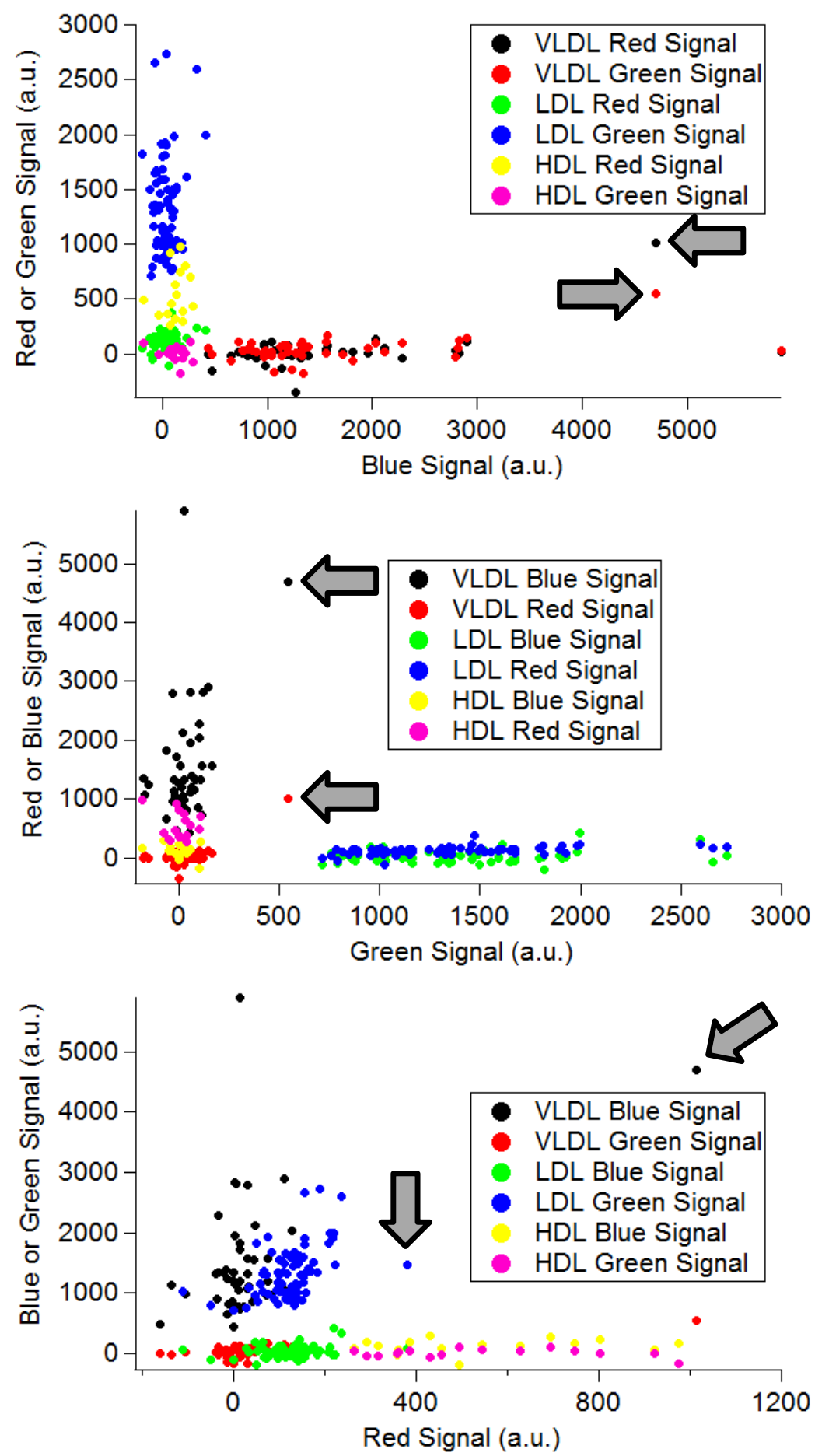

Figure S8. Donor C Fluorescent Signal Scatter Plots. Arrows mark apparent fusion particles. 

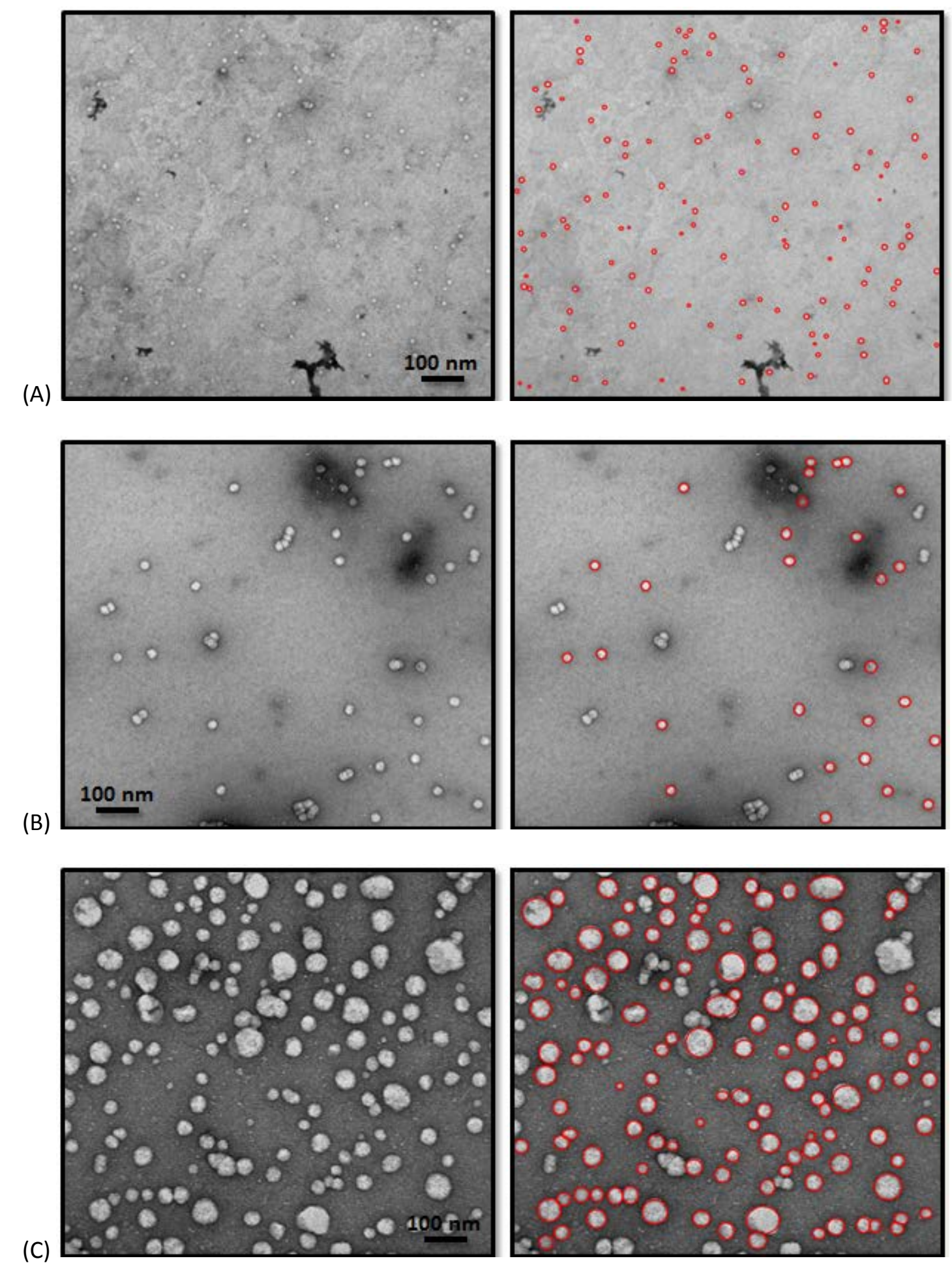

Figure S9. Donor A TEM Images. HDL labeled with Alexa 594 (A), LDL labeled with Alexa 488 (B), and VLDL labeled with Alexa 647 (C), before image processing (left) and after (right). For LDL, 2 images were used to generate the final histogram of particle sizes. 

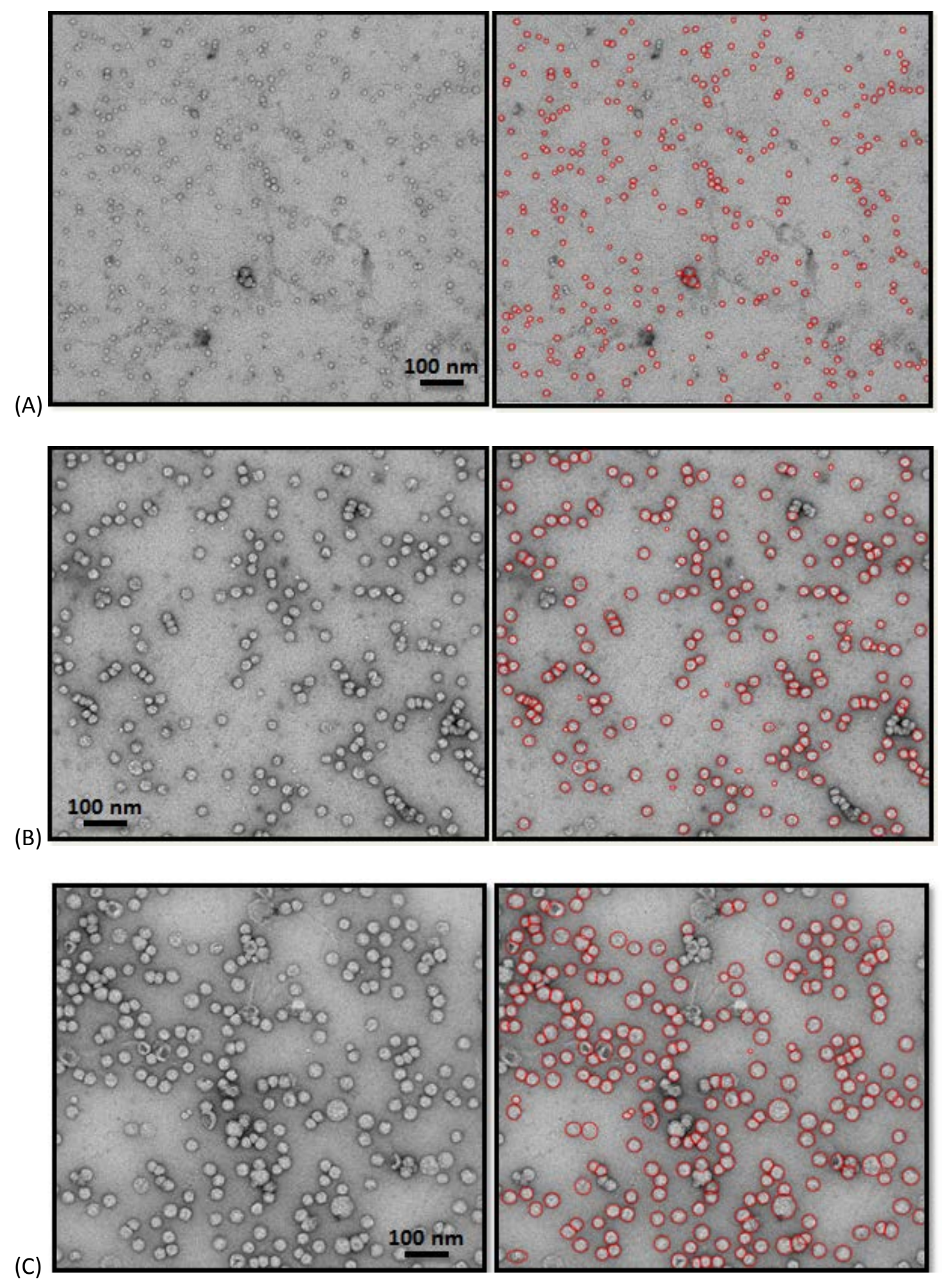

Figure S10. Donor B TEM Images. Unlabeled HDL (A), unlabeled LDL (B), and unlabeled VLDL (C), before image processing (left) and after (right). 

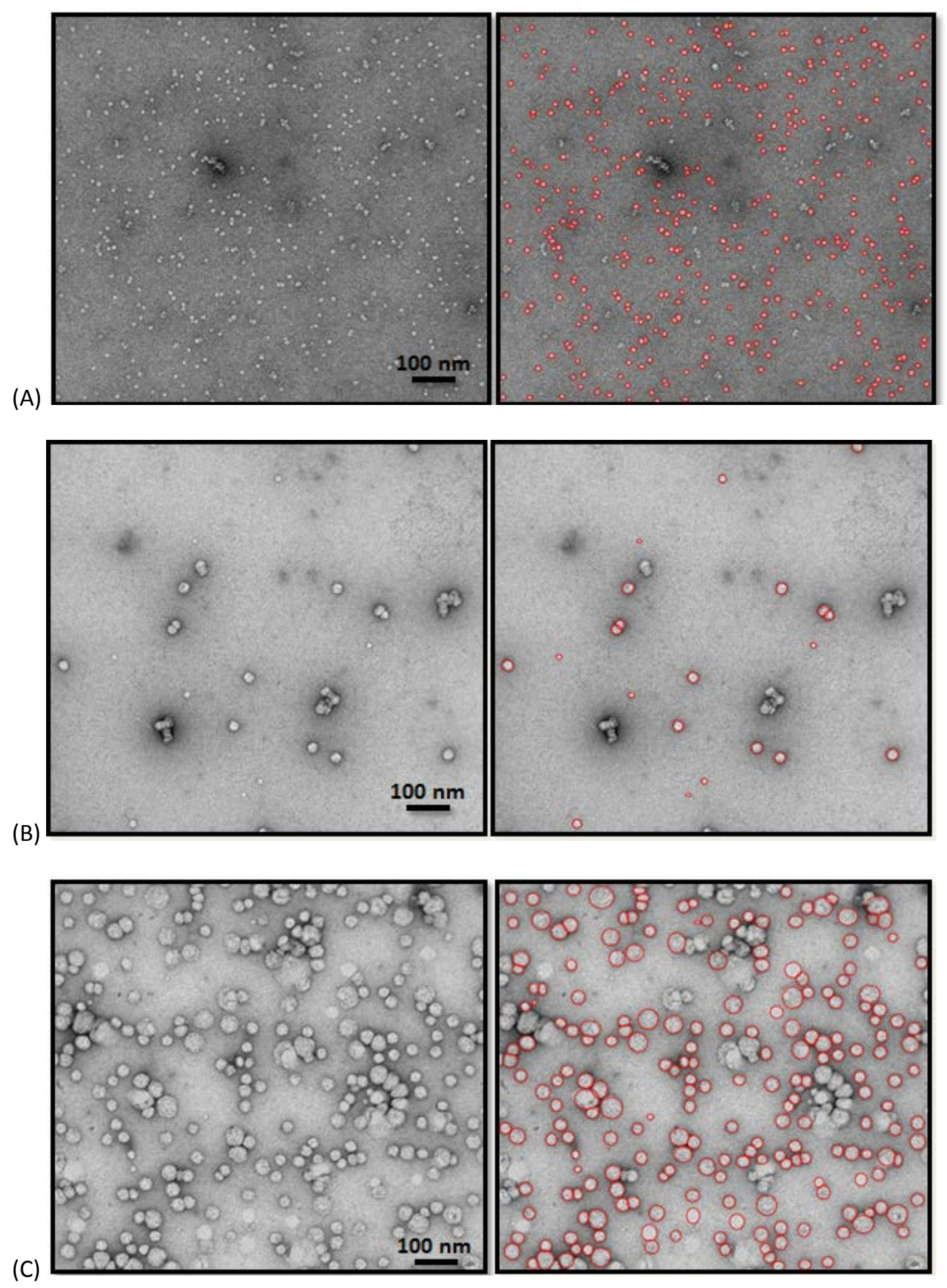

Figure S11. Donor C TEM Images. HDL labeled with Alexa 594 (A), LDL labeled with Alexa 488 (B), and VLDL labeled with Alexa 647 (C), before image processing (left) and after (right). For LDL, 10 images were used to generate the final histogram of particle sizes. 


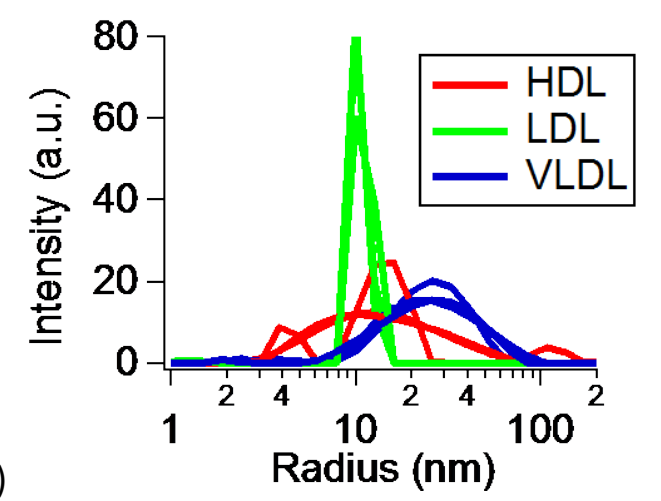

(A)

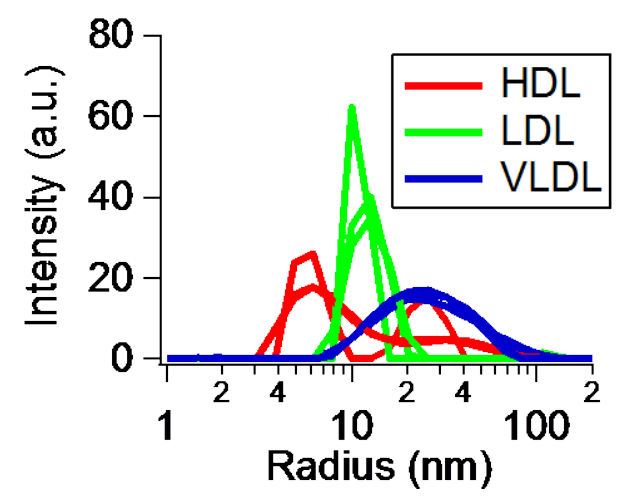

(B)

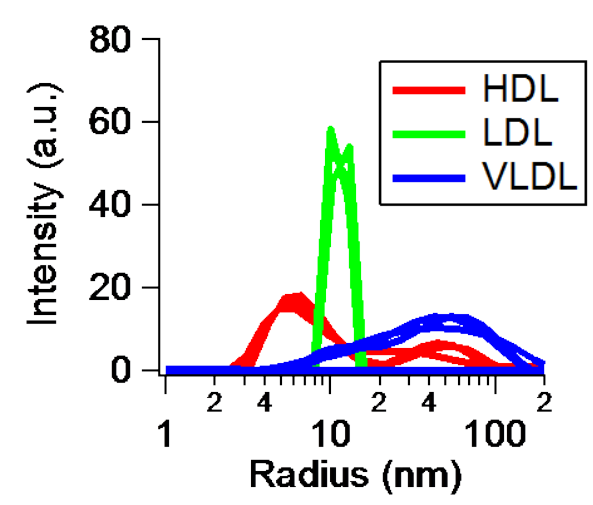

Figure S12. Dynamic Light Scattering (DLS) of HDL (red), LDL (green), and VLDL (blue) for Donor A (A), Donor B (B), and Donor $C$ (C). For each lipoprotein type, three results are shown to represent the fluctuations between measurements. DLS measurements were taken on a Dynapor Nanostar (Wyatt) at $100 \%$ power using 10 acquisitions of $5 \mathrm{~s}$ for each measurement. Unlabeled lipoproteins were diluted 1/10 into $\mathrm{NaPi}(50 \mathrm{mM}), \mathrm{NaCl}(150 \mathrm{mM}), \mathrm{EDTA}(0.5 \mathrm{mM}), \mathrm{pH} 7.4$ for measurement. The broader radial distribution observed for HDL can be attributed to a small number of larger particles in that fraction swamping the signal from the relatively small HDL. Larger particles are known to co-purify with the high density fraction from ultracentrifugation ${ }^{[4]}$. The LDL fractions had well-defined radial distributions with the expected radius of $\sim 10$ $\mathrm{nm}$. The broader distribution of VLDL is expected due to VLDL having an inherent polydisperse size distribution but this will also be biased towards the larger particles. 

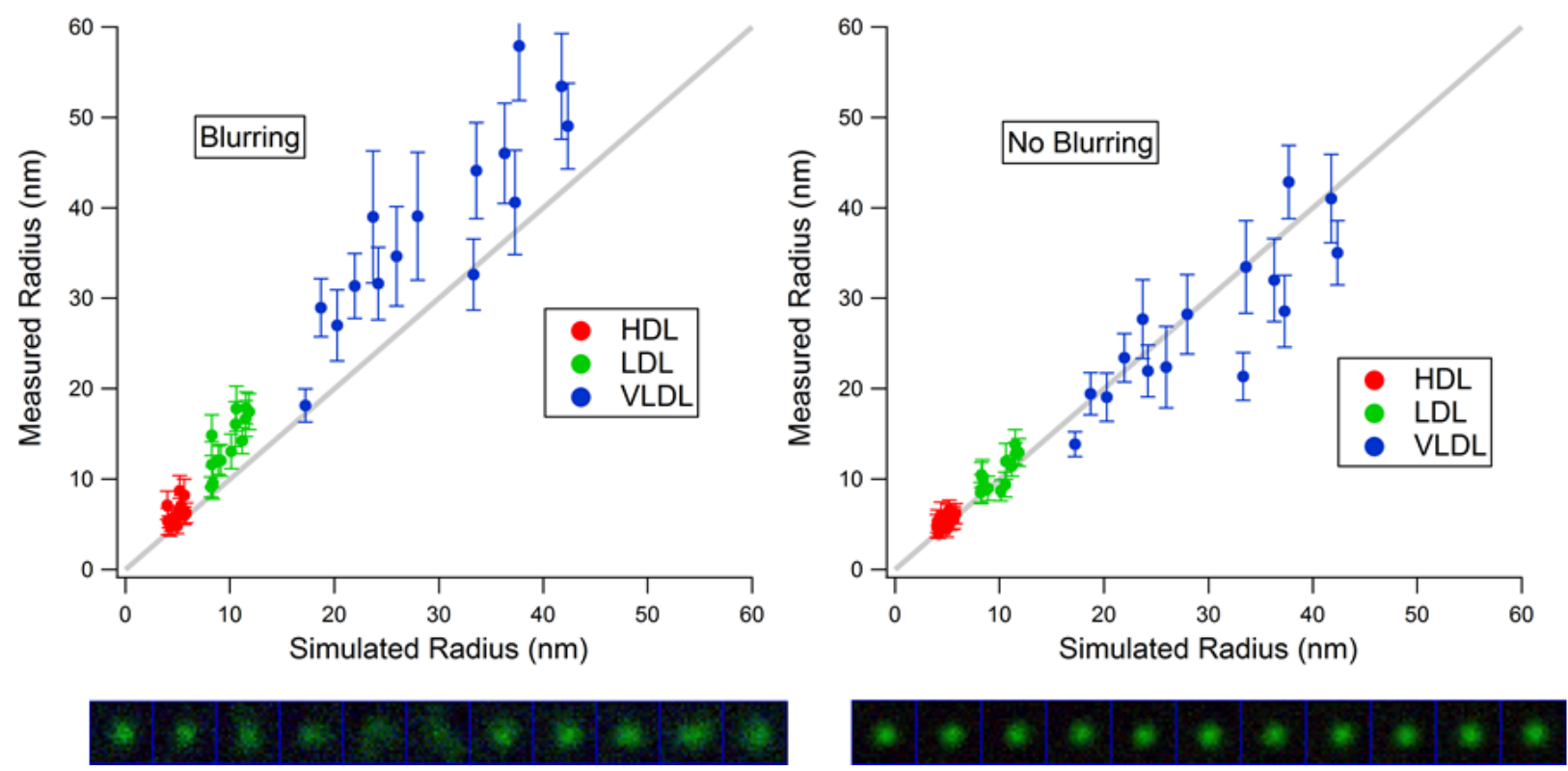

Figure S13. Simulated Data. Simulations with blurring over the $5 \mathrm{~ms}$ exposure time (left) and no blurring (right). The reference line (gray) plots $y=x$. The particles are from the provided movie Simulated Corrected.avi. Details of 20 particle tracks are provided in Simulated Particle Tracks. We ran simulations of mixed HDL, LDL, and VLDL and generated threechannel movies in the same format collected by the microscope. For each simulation, the particles were randomly assigned to be HDL, LDL, or VLDL and the radii were set as a linear random spread of 4-6 nm, 8-12 nm, and 15-45 nm, respectively. Half of the particles were designated as stuck to the surface. We did not simulate drag effects from the confining walls or non-specific interactions, so the only error in the determination of the radius will come from blurring of the particle image, since they move during the $5 \mathrm{~ms}$ exposure time. To investigate this blurring effect, we generated simulated images where the exposure time was instantaneous and unrealistic, as well as the more realistic case where the exposure was spread out over $5 \mathrm{~ms}$. For the case without blurring, no error was found between the true radius and the measured radius. For the blurring case, a shift towards larger radius was observed. Note that both cases were analyzed for the same simulation and same length of particle tracks. The only difference is that the blurring case introduces more uncertainty in the particle position, which results in an increased measured radius. We added noise to the background, uncertainty in the number of fluorophores, and bleaching effects. We did not analyze all tracks to the full $\mathbf{2 0 0}$ frames to better simulate real effects that cause some particles to produce longer tracks than others. The uncertainty in radius shown in the plots is a function of the number of frames analyzed for each track. Analytical results have been derived for blurring effects ${ }^{[5]}$. For the blurring simulations, the ratio of measured to true radius for HDL, LDL, and VLDL averaged to $1.27 \pm 0.06,1.41 \pm 0.05$, and $1.31 \pm 0.05$, respectively. Without blurring, the ratio of measured to true radius for HDL, LDL, and VLDL averaged to $1.10 \pm 0.04,1.08 \pm$ 0.04 , and $0.93 \pm 0.04$, respectively.

\section{References:}

[1] T. S. Kahlon, L. A. Glines, F. T. Lindgren, in Methods Enzymol. Vol. 129, Academic Press: New York, 1986, 26-45.

[2] Hoang, Nanotechnology 2011, 22.

[3] R. E. Morton, T. A. Evans, Anal. Biochem. 1992, 204, 332-334.

[4] Y. Yuana, J. Levels, A. Grootemaat, A. Sturk, R. Nieuwland, J. of Extracellular Vesicles 2014, 3.

[5] A. J. Berglund, Physical Review E 2010, 82. 